\title{
Dynamical conductivity of gated AA-stacking multilayer graphene with spin-orbital coupling
}

\author{
Cheng-Peng Chang *
}

June 3, 2022

\begin{abstract}
An efficient method with no numerical diagonalization of a huge Hamiltonian matrix and calculation of a tedious Green's function is proposed to acquire the exact energy spectrum and dynamical conductivity in a gated AA-stacking $N$-layer Graphene (AANLG) with the intrinsic spin-orbital coupling (SOC). $2 N \times 2 N$ tight-binding Hamiltonian matrix, velocity operator and Green's function representation of an AANLG are simultaneously reduced to $N$ $2 \times 2$ diagonal block matrices through a proper transformation matrix. A gated AANLG with intrinsic SOC is reduced to $N$ graphene-like layers. The energy spectrum of a graphene-like layer is $E=\varepsilon_{\perp} \pm \varepsilon_{\|} . \varepsilon_{\perp}$ depends on the interlayer interaction, gated voltage and layer number. $\varepsilon_{\|}=\sqrt{E_{M G}^{2}+\Delta^{2}}$, where $E_{M G}$ is the energy spectrum of a monolayer graphene and $\Delta$ is the magnitude of intrinsic SOC. More importantly, by inserting the diagonal block velocity operator and Green's function representation in the Kubo formula, the exact dynamical conductivity of an AANLG is shown to be $\sigma=\Sigma_{j=1}^{N} \sigma_{j}$, the sum of the dynamical conductivity of $N$ graphene-like layers. The analytical form of $\sigma_{j}$ is presented and the dependence of $\sigma_{j}$ on $\varepsilon_{\perp}, \Delta$, and chemical potential is clearly demonstrated. Moreover, the effect of Rashba SOC on the electronic properties of an AANLG is explored with the exact energy spectrum presented.
\end{abstract}

${ }^{*}$ Center for General Education, Tainan University of Technology, 710 Tainan, Taiwan E-mail: t00252@mail.tut.edu.tw 


\section{Introduction}

Graphene and its family members, including the AA-, AB- and ABC-stacking graphenes, have long attracted a lot of attention due to their striking physical properties. Graphene, a pure twodimensional (2D) system, is an atomic sheet peeled off from graphite[1, 2]. Carbon atoms are brought together and packed into a hexagonal lattice to form a graphene sheet. Such a geometrical structure consequently brings about a pair of low-lying linear energy bands. Electrons on the graphene sheet behave like the relativistic massless particles. This linear dispersion induces a variety of unique electronic properties, such as, electron-hole symmetry, Klein tunneling, high mobility at room temperature, non-zero conductivity, and anomalous quantum Hall effect [3, 4, 5, 6, 17, 8, 9, 10, 11, 12, 13, 14, 15, 16, 17]. Owing to the manifestation of fascinating effects, graphene is a promising material expected to play a vital role in technological applications, e.g., display screens, electric circuits, solar cells, analog electronics and photonics/optoelectronics[18, $19,20,21,22,23,24,25]$.

Multilayer graphenes are the pile of several graphene layers held together by the van der Waals force. The low-energy physical properties depend strongly both on the stacking order and on the number of layers[26, 27, 28, 29, 30, 31]. The most studied multilayer graphenes are $\mathrm{AB}$-stacking bilayer graphene[32, 33, 34, 35, 36, 37, 38, 39, 40] and ABC-stacking trilayer graphene [41, 42, 43, 44]. The AB-stacking bilayer graphene shows four parabolic bands around the Dirac points. The touch between valence and conduction bands makes the AB-stacking bilayer graphene a zero gap semimetal. A band gap is opened by the application of a vertical electric field[40]. The low energy dispersions of ABC-stacked trilayer graphene are described by two remarkably flat bands. The two-fold degeneracy in the band structure can be readily lifted by a perpendicular electrical field. Due to the progress in the fabrication and manipulation of graphene layers, the AA-stacking graphite and AA-stacking multilayer graphenes are produced[45, 46]. Following this, theoretical and experimental studies are conducted in order to explore electronic properties of AA-stacking bilayer and multilayer graphenes, e.g. infrared spectra, Raman spectra, Landau-level energies, absorption spectra, magneto absorption spectra, static polarization, and dynamical conductivity [45, 46, 47, 48, 49, 50, 51, 52, 53].

The increase in the layer number, appearance of interlayer interaction, and application of 
external field will lead to more difficulties exploring the electronic properties of the multilayer graphenes. For instance, a $2 N \times 2 N$ tight-binding (TB) Hamiltonian matrix is constructed and used to describe an $N$-layer graphene with the nearest neighbor interactions taken into account. The exact diagonalization of a $2 N \times 2 N$ Hamiltonian matrix will be cumbersome with the layer number $N$ increasing. A high-rank Hamiltonian matrix gives rise to more tasks in calculation of the Green's function, which is generally adopted to study the minimal and dynamical conductivities. Moreover, the mirror-symmetry-breaking, caused by a vertical electric field applied to a multilayer graphene, also increases the difficulty in the diagonalization of Hamiltonian matrix. Most of studies focus on the exploration of physical properties as the layer number $N<3$, e. g., $\mathrm{AB}$-stacking bilayer graphene, and $\mathrm{ABC}$-stacking trilayer graphenes. Recently, the investigations of the dynamical conductivity of AA-stacking graphene and static polarization of AAA-stacking graphene have been reported [52, 53]. A model can deal with physical properties of multilayer graphene in different stacking order or various layer number $N$ under external field is inspired and desired. We previously presented analytical modes to exactly describe the minimal conductivity of the AB-stacking multilayer graphene [54] and exact Landau levels of the AA-stacking multilayer graphene[55].

In this work, an analytical model is proposed in order to derive the dynamical conductivity and energy spectrum in a gated AANLG with intrinsic SOC. $2 N \times 2 N$ Hamiltonian matrix of AANLG is decomposed into $N 2 \times 2$ diagonal block matrices. An AANLG is decoupled into $N$ graphene-like layers. Thus, a close form of the energy spectrum is disposed. Application of current analytical model to the study of the dynamical conductivity of AANLG is conducted. It is shown that the dynamical conductivity of an AANLG is equal to the sum of the dynamical conductivity of $N$ graphene-like layers with/without intrinsic SOC. Above all, the presented model can efficiently and exactly give out the energy spectrum and dynamical conductivity in a gated AANLG with intrinsic SOC and avoid the diagonalization of a huge Hamiltonian matrix and calculation of associated Green's function. 


\section{Gate-Tuned Energy Spectrum Of AANLG with Spin-Orbital Coupling}

Graphene is a two dimensional atomic sheet made up of carbon atoms, which are precisely packed in a planar hexagonal lattice, viewed as bipartite lattice composed of two interpenetrating triangular sublattices. The carbon-carbon bond length is $b=1.42$ and the lattice vector is equal to $a=\sqrt{3} b$. A primitive cell contains two atoms denoted as $A$ and $B$. With SOC Taken into consideration, the Hamiltonian $H_{M G}$ of a monolayer graphene is [56, 57]

$$
H_{M G}=h_{0}+h_{I S O}+h_{R}
$$

where the first term, $h_{0}=\sum_{i, j} \alpha_{0} c_{i}^{+} c_{j}+h . c$. , is Hamiltonian operator of the monolayer graphene without SOC. $c_{i}^{+}\left(c_{j}\right)$ is the creation (annihilation) operator and creates (annihilates) an electron at the site $i(j) . \alpha_{0}$ is the intralayer nearest-neighbor hopping between atoms $A$ and $B$ on the same graphene layer, as illustrated in Fig. 1. The second term $h_{I S O}$ is the intrinsic spin-orbit interaction. The third term is the Rashba SOC, which is induced by the external perpendicular electric field or the interaction with substrate. The Hamiltonian operator of Rashba SOC is $h_{R}=$ $-i \lambda_{R} \sum_{\langle i, j\rangle} \sum_{\mu, v} c_{i}^{+}\left(\mathbf{S}_{\mu, v} \times d_{i, j}\right)_{z} c_{j}+h . c .$, where $\lambda_{R}$ is the magnitude of the Rashba SOC. $\mathbf{S}$ is the Pauli vector, the subscripts $\mu$ and $v$ represent the spin index, and $d_{i, j}$ is the unit vector pointing from atom site $i$ to its nearest neighbor $j$.

Without the Rashba SOC $\left(\lambda_{R}=0\right)$, the TB Hamiltonian matrix, spanned by periodic Bloch functions $|A\rangle$ and $|B\rangle$, is [52]

$$
H_{M G}=\left(\begin{array}{cc}
\Delta \tau_{z} s_{z} & \alpha_{\mathbf{k}} \\
\alpha_{\mathbf{k}}^{*} & -\Delta \tau_{z} s_{z}
\end{array}\right)
$$

where $\alpha_{\mathbf{k}}=\alpha_{0} f(\mathbf{k})=\alpha_{0} \sum_{j=1}^{3} \exp \left(i \mathbf{k} \cdot \mathbf{b}_{j}\right) . \quad \mathbf{b}_{j}$ represents the three nearest neighbors on the same graphene plane and $\mathbf{k}$ is the in-plane wave vector. $\Delta$ is the strength of ISOC and $s_{z}= \pm 1$ represents the up or down spin. $\tau_{z}= \pm 1$ at the Dirac points $\mathrm{K}$ and $\mathrm{K}$ '. The energy dispersions are $E= \pm \sqrt{\left|\alpha_{\mathbf{k}}\right|^{2}+\Delta^{2}}$

Furthermore, the TB Hamiltonian matrix of the Hamiltonian $H_{M G}=h_{0}+h_{I S O}+h_{R}$, acting 
on periodic Bloch functions $|A \uparrow\rangle,|B \uparrow\rangle,|A \downarrow\rangle,|B \downarrow\rangle$, is[57]

$$
H_{M G}=\left(\begin{array}{cccc}
\Delta & \alpha_{\mathbf{k}} & 0 & 0 \\
\alpha_{\mathbf{k}}^{*} & -\Delta & -i \lambda_{R} & 0 \\
0 & i \lambda_{R} & -\Delta & \alpha_{\mathbf{k}} \\
0 & 0 & \alpha_{\mathbf{k}}^{*} & \Delta
\end{array}\right)
$$

where the Rashba SO interaction between $|A \uparrow\rangle$ and $|B \downarrow\rangle(|B \uparrow\rangle$ and $|A \downarrow\rangle)$ is neglected because it is much weaker than $\lambda_{R}[57]$. The analytical energy dispersions are

$$
\Lambda_{ \pm \pm}= \pm \frac{\lambda_{R}}{2} \pm \sqrt{\left|\alpha_{\mathbf{k}}\right|^{2}+\Delta^{2}+\lambda_{R} \Delta+\frac{\lambda_{R}^{2}}{4}}
$$

\subsection{Energy Spectrum Of AANLG with Intrinsic SOC}

By stacking $N$ layer graphenes directly on each other with an interlayer distance between graphenes $c=3.35 \AA[58]$, an AANLG is formed, as shown in Fig. 1. In the stacking direction, $N$ atoms $A$ (B) form a linear chain. The primitive unit cell contains $2 N$ atoms, denoted as $A_{1}, A_{2}, \cdots A_{N}, B_{1}, B_{2}, \cdots, B_{N}$, The first Brillouin zone is the same as that of a graphene. The Hamiltonian of an AANLG is given by

$$
H_{A A N L A}=H_{0}+H_{I S O}+H_{R}
$$

where $H_{0}$ is Hamiltonian operator an AANLG without SOC, and $H_{I S O}$ and $H_{R}$ are caused by ISO and Rashba SO interactions. Following the discussion in the subsection above, we first close the Rashba effect, i. e. , $H_{R}=0$. In the presence of an electric field, the Hamiltonian representation of an AANLG with intrinsic SOC, spanned by periodic Bloch functions $\left|A_{1} \uparrow\right\rangle,\left|A_{2} \uparrow\right\rangle, \cdots\left|A_{N} \uparrow\right\rangle$, $\left|B_{1} \uparrow\right\rangle,\left|B_{2} \uparrow\right\rangle, \cdots\left|B_{N} \uparrow\right\rangle$, is a $2 N \times 2 N$ matrix, reading

$$
H=\left(\begin{array}{ll}
H_{A A} & H_{A B} \\
H_{B A} & H_{B B}
\end{array}\right),
$$


where $H_{A A}, H_{A B}, H_{B A}$, and $H_{B B}$ are $N \times N$ matrices. The Hamiltonian operators $H_{A A}$ and $H_{A B}$ describe an $N$-site linear chain with the intrinsic SOC subjected to a parallel electric field.

The recent researches[59, 60], using ab intial calculation and TB method, show that in the Bernal stacking bilayer graphene and $\mathrm{ABC}$ stacking trilayer graphene, many intralayer and interlayer SOC parameters are included to fully describe the spin-orbital interactions in the TB model. The numerical fitting of TB parameters to the results of ab initial calculation exhibits that the magnitudes of intrinsic $(\Delta)$ and Rashba spin-orbital interactions $\left(\lambda_{R}\right)$ are layer-positiondependent. What's more, the strength of the interlayer intrinsic SOC is much weaker than that of the intralayer intrinsic SOC. Accordingly, we neglect the interlayer intrinsic spin-orbital interactions in our case and then take the intralayer intrinsic and Rashba spin-orbital interactions into account. Thus, the two $4 \times 4 H_{A A}$ and $H_{B B}$ Hamiltonian matrices of the AA-stacking quad-layer graphene, for instance, are expressed as the following,

$$
\begin{gathered}
H_{A A}=\left(\begin{array}{cccc}
3 V / 2+\Delta_{1} & \alpha_{1} & 0 & 0 \\
\alpha_{1} & V / 2+\Delta_{2} & \alpha_{1} & 0 \\
0 & \alpha_{1} & -V / 2+\Delta_{3} & \alpha_{1} \\
0 & 0 & \alpha_{1} & -3 V / 2+\Delta_{4}
\end{array}\right), \\
H_{B B}=\left(\begin{array}{cccc}
3 V / 2-\Delta_{1} & \alpha_{1} & 0 & 0 \\
\alpha_{1} & V / 2-\Delta_{2} & \alpha_{1} & 0 \\
0 & \alpha_{1} & -V / 2-\Delta_{3} & \alpha_{1} \\
0 & 0 & \alpha_{1} & -3 V / 2-\Delta_{4}
\end{array}\right),
\end{gathered}
$$

where $\Delta_{j}(j=1,2,3$ and 4$)$ is the intralayer intrinsic spin-orbital interaction of the $j$-layer graphene. $V=|e| F c$ is the effect electric potential difference between the adjacent layers caused by the external electric field. Then, $V$ is denoted as the gate voltage. In multilayer graphenes, the potential drop between two adjacent layers might be affected by the screening processes[61]. For simplicity, we also assume that the potential drop is the same on each graphene layer. The interlayer hopping parameter, $\alpha_{1}$, couples the two $A$ (or $B$ ) atoms from the two adjacent layers [Fig. 1]. $\alpha_{3}$, the interlayer interaction between atoms $A$ and $B$ from the two adjacent layers, results 
in a weak electron-hole asymmetry in an AANLG [55, 58]. The values of the hopping integrals are $\alpha_{1}=0.361 \mathrm{eV}$ and $\alpha_{3}=-0.032 \mathrm{eV}$ [58]. Only the main interlayer interaction $\alpha_{1}$ is taken into consideration because of $\alpha_{3} \ll \alpha_{1}$. The matrix element $H_{A B}$, resulting from the intralayer interaction, reads

$$
H_{A B}=H_{A B}^{*}=\left(\begin{array}{cccc}
\alpha_{\mathbf{k}} & 0 & 0 & 0 \\
0 & \alpha_{\mathbf{k}} & 0 & 0 \\
0 & 0 & \alpha_{\mathbf{k}} & 0 \\
0 & 0 & 0 & \alpha_{\mathbf{k}}
\end{array}\right)
$$

The layer-position-dependent intralayer intrinsic spin-orbital interaction and Rashba SOC destroy the inversion symmetry of AANGL. The breaking of the inversion symmetry complicates the analysis and discussion. For simplicity, the intralayer intrinsic spin-orbital interaction are assumed to be independent of the vertical positions; that is, $\Delta_{j}=\Delta$ and $\lambda_{R, j}=\lambda_{R}$. Notably, the $H_{A A}\left(H_{B B}\right)$ is the sum of two matrices $H_{V}$ and $H_{I S O}$ and reads

$$
\begin{aligned}
& H_{A A}=H_{V}+H_{I S O}=H_{V}+\Delta \mathbb{1}, \\
& H_{B B}=H_{V}+H_{I S O}=H_{V}-\Delta \mathbb{1},
\end{aligned}
$$

where $\mathbb{1}$ is a $N \times N$ identity matrix. $H_{V}$ describes an $N$-site linear chain without SOC subjected to a parallel electric field. $H_{A A}\left(H_{B B}\right)$ is commute with $H_{V}$; that is, $H_{A A}\left(H_{B B}\right)$ and $H_{V}$ share the same eigenfunctions. The eigenenergy $\varepsilon_{j}$ and associated eigenfunction $\left|\mathrm{S}_{j}\right\rangle$ of $H_{V}$ are easily obtained through the diagonalization of the eigenvalue equation[55]

$$
H_{V}\left|\mathrm{~S}_{j}\right\rangle=\varepsilon_{j}\left|\mathrm{~S}_{j}\right\rangle
$$

where $j=1,2, \cdots, N$. The transpose of $\left|\mathrm{S}_{j}\right\rangle$ is $\left|\mathrm{S}_{j}\right\rangle^{T}=\left|s_{j, 1}, s_{j, 2}, s_{j, 3}, \cdots s_{j, N}\right\rangle$ and the component $s_{j, l}$, is the site amplitude of atom $A$ or $B$ located at the $l$ th layer.

With column vectors $\left|\mathrm{S}_{j}\right\rangle$, the eigenfunctions, an $N \times N$ unitary transformation matrix $\hat{U}_{V}=$ $\left(\left|\mathrm{S}_{1}\right\rangle,\left|\mathrm{S}_{2}\right\rangle, \cdots,\left|\mathrm{S}_{N}\right\rangle\right)$ is then constructed and used to diagonalize $H_{V}$, i. e., $\hat{U}_{V}^{\dagger} H_{V} \hat{U}_{V}=\varepsilon_{j} \mathbb{1}$, 
where $\mathbb{1}$ is a unit matrix. The eigen-energies of $H_{A A}$ and $H_{B B}$ are $\varepsilon_{j}+\Delta$ and $\varepsilon_{j}-\Delta$ after the diagonalization of Eqs. (7a) and (7b), respectively .

To acquire the energy spectrum of AANLG, a $2 N \times 2 N$ unitary transformation matrix

$$
\mathbf{U}=\left(\begin{array}{cc}
\hat{U}_{V} & 0 \\
0 & \hat{U}_{V}
\end{array}\right)
$$

is built to transform the Hamiltonian matrix (Eq. (6a) ) into a simple form. After the unitary transformation, a reduced matrix $\mathscr{H}_{\text {red }}=\mathbf{U}^{\dagger} H \mathbf{U}$ has the form

$$
\mathscr{H}_{\text {red }}=\left(\begin{array}{cc}
\left(\varepsilon_{j}+\Delta\right) \mathbb{1} & \alpha_{\mathbf{k}} \mathbb{1} \\
\alpha_{\mathbf{k}}^{*} \mathbb{1} & \left(\varepsilon_{j}-\Delta\right) \mathbb{1}
\end{array}\right),
$$

where $\mathbb{1}$ is $N \times N$ unit matrix. Then, the reduced Hamiltonian matrix can be rearranged into block diagonal form, $\mathscr{H}_{\text {red }}=\mathscr{H}_{1} \oplus \mathscr{H}_{2} \oplus \cdots \oplus \mathscr{H}_{N}$, where each $2 \times 2$ block diagonal matrix $\mathscr{H}_{j}$ is expressed as follows

$$
\mathscr{H}_{j}=\left(\begin{array}{cc}
\varepsilon_{j}+\Delta & \alpha_{\mathbf{k}} \\
\alpha_{\mathbf{k}}^{*} & \varepsilon_{j}-\Delta
\end{array}\right)
$$

That is to say, an AANLG can be decomposed into $N$ subsystems, $\mathscr{H}_{j}$. The exact energy spectrum of each subsystem is

$$
E_{j, \pm}=\varepsilon_{j} \pm \sqrt{\left|\alpha_{\mathbf{k}}\right|^{2}+\Delta^{2}}=\varepsilon_{\perp} \pm \varepsilon_{\|}
$$

where $\varepsilon_{j}\left(=\varepsilon_{\perp}\right)$ depends on the magnitude of interlayer interaction, gated voltage and layer number. $\varepsilon_{||}=\sqrt{\left|\alpha_{\mathbf{k}}\right|^{2}+\Delta^{2}}$ is the energy spectrum of a monolayer graphene with SOC.

Around the Dirac point $K$, the diagonal block is for $\mathbf{k}=\mathbf{K}+\mathbf{q}$

$$
\mathscr{H}_{j}=\left(\begin{array}{cc}
\varepsilon_{j}+\Delta & -\hbar v_{F}\left(q_{x}+i q_{y}\right) \\
-\hbar v_{F}\left(q_{x}-i q_{y}\right) & \varepsilon_{j}-\Delta
\end{array}\right)
$$

and $\hbar v_{F}=\frac{3}{2} \alpha_{0} b$ is the Fermi velocity. The low-lying energy dispersions associated with $\mathscr{H}_{j}$ are $E_{j, \pm}=\varepsilon_{j} \pm \sqrt{\left|\hbar v_{F} q\right|^{2}+\Delta^{2}}$, where $|q|=\sqrt{q_{x}^{2}+q_{y}^{2}}$. 


\subsection{Energy Spectrum Of AANLG with Intrinsic and Rashba spin-orbital Interactions}

If we take both the intrinsic and Rashba spin-orbital interactions into consideration, TB Hamiltonian matrix of an AANLG subject to a perpendicular electric field, acting on periodic Bloch functions $\left|A_{1} \uparrow\right\rangle,\left|B_{1} \uparrow\right\rangle,\left|A_{1} \downarrow\right\rangle,\left|B_{1} \downarrow\right\rangle, \cdots,\left|A_{j} \uparrow\right\rangle,\left|B_{j} \uparrow\right\rangle,\left|A_{j} \downarrow\right\rangle,\left|B_{j} \downarrow\right\rangle, \cdots,\left|A_{N} \uparrow\right\rangle,\left|B_{N} \uparrow\right\rangle, \mid A_{N} \downarrow$ \rangle,$\left|B_{N} \downarrow\right\rangle$, is a $4 N \times 4 N$ Hermitian matrix and expressed as follows

$$
H_{A A N L G}=\left(\begin{array}{cccccc}
H_{1} & H_{T} & 0 & \cdots & \cdots & 0 \\
H_{T} & H_{2} & H_{T} & \ddots & \vdots & 0 \\
0 & H_{T} & H_{3} & H_{T} & 0 & \cdots \\
0 & 0 & \ddots & \ddots & \ddots & \vdots \\
0 & \vdots & \ddots & \ddots & \ddots & H_{T} \\
0 & \cdots & 0 & \cdots & H_{T} & H_{N}
\end{array}\right),
$$

where $H_{j}$ and $H_{T}$ are $4 \times 4$ blocks. The off-diagonal block $H_{T}=\alpha_{1} \mathbb{1}$ originates in the main interlayer interaction $\alpha_{1}$. The diagonal block $H_{j}=V_{j} \mathbb{1}+H_{M G}$ is the Hamiltonian matrix of the $j$-layer graphene in the presence of the gated potential $V_{J}$, which has the form

$$
H=\left(\begin{array}{cccc}
V_{j}+\Delta & \alpha_{\mathbf{k}} & 0 & 0 \\
\alpha_{\mathbf{k}}^{*} & V_{J}-\Delta & -i \lambda_{R} & 0 \\
0 & i \lambda_{R} & V_{J}-\Delta & \alpha_{\mathbf{k}} \\
0 & 0 & \alpha_{\mathbf{k}}^{*} & V_{J}+\Delta
\end{array}\right)
$$

It is easy to diagonalize the block $H_{j}=V_{j} \mathbb{1}+H_{M G}$ through a $4 \times 4$ unitary transformation matrix $\mathbb{U}$, which transforms $H_{M G}$ into a diagonal matrix, .i. e., $\mathbb{U}^{+} H_{M G} \mathbb{U}=\operatorname{diag}\left(\Lambda_{++}, \Lambda_{+-}, \Lambda_{--}, \Lambda_{-+}\right)$ (Eq. (4)). The eigenvalues of $H_{j}$ are $\Lambda_{J, \pm \pm}=V_{J}+\Lambda_{ \pm \pm}$. Then, a $4 N \times 4 N$ unitary transformation matrix $\mathbf{U}=\operatorname{diag}(\mathbb{U}, \mathbb{U}, \ldots, \mathbb{U})$ is constructed and used to transform $H_{A A N L G}$ into a diagonal block form. After the operation, we obtain $\mathbf{U}^{+} H_{A A N L G} \mathbf{U}=H_{++} \oplus H_{+-} \oplus H_{--} \oplus H_{-+}$, where $\mathscr{H}_{\eta \xi}$ ( $\eta= \pm, \xi= \pm)$ is an $N \times N$ matrix.

We take the AA-stacking trilayer graphene as a study model. The Hamiltonian matrix $H_{A A T L G}$ 
and unitary transform matrix $\mathbf{U}$ are

$$
H_{A A T L G}=\left(\begin{array}{ccc}
H_{1} & H_{T} & 0 \\
H_{T} & H_{2} & H_{T} \\
0 & H_{T} & H_{3}
\end{array}\right), \text { and } U=\left(\begin{array}{ccc}
\mathbb{U} & 0 & 0 \\
0 & \mathbb{U} & 0 \\
0 & 0 & \mathbb{U}
\end{array}\right)
$$

After the operation $\mathbf{U}^{+} H_{A A T L G} \mathbf{U}, H_{A A T L G}$ is arranged into the block diagonal form $H_{A A T L G}=$ $H_{++} \oplus H_{+-} \oplus H_{--} \oplus H_{-+}$, where each $H_{\eta \xi}$ is $3 \times 3$ matrix and $H_{\eta \xi}=\Lambda_{\eta \xi} \mathbb{1}+H_{V}$. The latter term $H_{V}$, Eq. $(7 \mathrm{a})$, describes an $N(=3)$-site linear chain without SOC subjected to a parallel electric field. The energy spectrum related to $H_{\eta \xi}$ are $E=\Lambda_{\eta \xi}+\varepsilon_{j}=\varepsilon_{||}+\varepsilon_{\perp}$, where $\Lambda_{\eta \xi}=\varepsilon_{\|}$ and $\varepsilon_{\perp}=\sqrt{V^{2}+2 \alpha_{1}^{2}}, 0$, or $-\sqrt{V^{2}+2 \alpha_{1}^{2}}[55]$.

\section{Electronic Properties and Discussions}

The energy dispersions of an AANLG with SOC in the presence of the gated potential are easily obtained through the calculation of energy spectrum of each subsystem by using analytical formula $E_{j}=\varepsilon_{\perp}+\varepsilon_{\|}$. For example, the energy spectrum of the AA-stacking bilayer graphene are described as $E= \pm \sqrt{\alpha_{1}^{2}+v^{2} / 4}+\Lambda_{ \pm \pm}$, where $\Lambda_{ \pm \pm}= \pm \frac{\lambda_{R}}{2} \pm \sqrt{\left|\alpha_{\mathbf{k}}\right|^{2}+\Delta^{2}+\lambda_{R} \Delta+\frac{\lambda_{R}^{2}}{4}}$. Since the energy dispersions are symmetry about $E=0$, only the energy spectrum $E>0$ are shown in Fig. 2. In the absence of the gates potential and SOC, the energy dispersions around the Dirac point $\mathbf{K}$ illustrate one pair of linear bands crossing at $E=\alpha_{1}$ (dashed curves in the inset). The gated potential $V=0.4 \alpha_{1}$ shifts the linear bands upward (red solid curves in the inset). In the AB-stacking bilayer graphene, the intrinsic SOC parameter is $\Delta \sim 10^{-5} \mathrm{eV}$ and hence $\Delta / \alpha_{1} \sim 10^{-4}$ [59]. For convenience of numerical analysis, we use $\Delta / \alpha_{1}=0.1$ in this work. The analytical model and numerical results are relevant and applicable to the exploration of physical properties in multilayer graphene-like systems. The inclusion of the intrinsic SOC $\Delta=0.1 \alpha_{1}$ changes the linear bands (red solid curves) into the parabolic bands (blue solid curves), which are described by $E=\sqrt{\alpha_{1}^{2}+v^{2} / 4}+\sqrt{\left|\hbar v_{F} q\right|^{2}+\Delta^{2}}$. The maximum (minimum) of the parabolic band, located at the Dirac point $K$, is $E=(\sqrt{1.16}-0.1) \alpha_{1}\left(E=(\sqrt{1.16}+0.1) \alpha_{1}\right)$. The green curves are the energy spectrum of the gated AA-stacking bilayer graphene with the Rashba SO interaction $\lambda_{R}=0.05 \alpha_{1}$. The Rashba SO interaction $\lambda_{R}$ destroys the degeneracy of the linear 
bands and produces four parabolic bands (green curves). The middle two parabolic bands touch each other at $E=\sqrt{1.16} \alpha_{1}$.

The energy dispersions of the AA-stacking trilayer graphene are also evaluated with formula $E=\Lambda_{ \pm \pm}+\varepsilon_{j}$, where $\varepsilon_{1}=\sqrt{V^{2}+2 \alpha_{1}^{2}}, \varepsilon_{2}=0$, and $\varepsilon_{3}=-\sqrt{V^{2}+2 \alpha_{1}^{2}}$. Without SOC, the energy spectrum $E_{2}=\Lambda_{ \pm \pm}+\varepsilon_{2}$ are independent of the magnitude of gated potential, as shown by the black dashed and red solid curves in the inset of Fig. 3. Two linear bands cross over at $E=0$. The intrinsic SOC $\Delta$ changes the linear into the parabolic bands, as illustrated by dashed blue curves, which is simulated by $E_{2}=\sqrt{\left|\hbar v_{F} q\right|^{2}+\Delta^{2}}$, The maximum (minimum) of the parabolic band is determined by the strength of $\Delta=0.1 \alpha_{1}$. There are four parabolic bands after the inclusion of the Rashba SOC (green cures). The middle two parabolic bands do not touch at $E=0$ due to the intrinsic SOC.

\section{Green's function and Velocity operator}

After the introduction of the Rashba effect, as illustrated in the section above, the $4 N \times 4 N$ Hamiltonian matrix can be divided into four $N \times N$ diagonal blocks. This would complicate the discussion and block us to pursue a simple analytical form of the conductivity of an AANLG. We, then, switch off the Rashba effect and consider the intrinsic SOC (ISOC) alone in the following work. Now, we exhibit that the Green's function and velocity operator associated with an AANLG can be transformed into the diagonal block matrices. With the Hamiltonian matrix $H$, it is straightforward to calculate the Green's function through $G(z)=\frac{1}{z I-H}$. The larger Hamiltonian matrix gives rise to more complex tasks in calculation of the inverse matrix of $z I-H$. To reduce the task, we use the unitary operator $\mathbf{U}$, which causes $\mathscr{H}_{\text {red }}=\mathbf{U}^{\dagger} H \mathbf{U}$, to transform the Green's function. After the operation, we have $\mathscr{G}=\mathbf{U}^{\dagger} G \mathbf{U}=\mathbf{U}^{\dagger} \frac{1}{z I-H} \mathbf{U}=\frac{1}{z I-\mathscr{H}_{\text {red }}} . \mathscr{H}_{\text {red }}$ is a block diagonal matrix and so does $\left(z I-\mathscr{H}_{\text {red }}\right)$. Now, the Green's function $\mathscr{G}$ is also in a block diagonal form; that is, $\mathscr{G}=\mathscr{G}_{1} \oplus \mathscr{G}_{2} \oplus \cdots \oplus \mathscr{G}_{N}$. Moreover, each sub-Green's function is $\mathscr{G}_{j}=\frac{1}{z I-\mathscr{H}_{j}}$ and it is a $2 \times 2$ matrix,

$$
\mathscr{G}_{j}^{-1}=\left(\begin{array}{cc}
z-\varepsilon_{j}-\Delta & -\alpha_{\mathbf{k}} \\
-\alpha_{\mathbf{k}}^{*} & z-\varepsilon_{j}+\Delta
\end{array}\right) \text {, }
$$


with the corresponding elements

$$
\begin{aligned}
& \mathscr{G}_{j, 11}(z)=\frac{z-\varepsilon_{j}+\Delta}{\left(z-\varepsilon_{j}\right)^{2}-\left(\Delta^{2}+\left|\alpha_{\mathbf{k}}\right|^{2}\right)}=\frac{\frac{1}{2}\left[1+\frac{\Delta}{\sqrt{\Delta^{2}+\left|\alpha_{\mathbf{k}}\right|^{2}}}\right]}{z-E_{j,+}}+\frac{\frac{1}{2}\left[1-\frac{\Delta}{\sqrt{\Delta^{2}+\left|\alpha_{\mathbf{k}}\right|^{2}}}\right]}{z-E_{j,-}} \\
& \mathscr{G}_{j, 12}(z)=\frac{\alpha_{\mathbf{k}}^{*}}{\left(z-\varepsilon_{j}\right)^{2}-\left(\Delta^{2}+\left|\alpha_{\mathbf{k}}\right|^{2}\right)}=\frac{\alpha_{\mathbf{k}}^{*}}{2 \sqrt{\Delta^{2}+\left|\alpha_{\mathbf{k}}\right|^{2}}}\left[\frac{1}{z-E_{j,+}}-\frac{1}{z-E_{j,-}}\right] \\
& \mathscr{G}_{j, 21}(z)=\frac{\alpha_{\mathbf{k}}}{\left(z-\varepsilon_{j}\right)^{2}-\left(\Delta^{2}+\left|\alpha_{\mathbf{k}}\right|^{2}\right)}=\frac{\alpha_{\mathbf{k}}}{2 \sqrt{\Delta^{2}+\left|\alpha_{\mathbf{k}}\right|^{2}}}\left[\frac{1}{z-E_{j,+}}-\frac{1}{z-E_{j,-}}\right] \\
& \mathscr{G}_{j, 22}(z)=\frac{z-\varepsilon_{j}-\Delta}{\left(z-\varepsilon_{j}\right)^{2}-\left(\Delta^{2}+\left|\alpha_{\mathbf{k}}\right|^{2}\right)}=\frac{\frac{1}{2}\left[1-\frac{\Delta}{\sqrt{\Delta^{2}+\left|\alpha_{\mathbf{k}}\right|^{2}}}\right]}{z-E_{j,+}}+\frac{\frac{1}{2}\left[1+\frac{\Delta}{\sqrt{\Delta^{2}+\left|\alpha_{\mathbf{k}}\right|^{2}}}\right]}{z-E_{j,-}} .
\end{aligned}
$$

The velocity operator, $\mathbf{V}=\frac{\partial H}{\partial \hbar \mathbf{k}}$, is approximated as the derivative of the Hamiltonian with respect to the momentum $\hbar \mathbf{k}$, based on the gradient approximation. According to the Eq. 6a), the velocity matrix related to AANLG is

$$
\mathbf{V}=\left(\begin{array}{cc}
\frac{\partial H_{A A}}{\partial \mathbf{k}} & \frac{\partial H_{A B}}{\partial \mathbf{k}} \\
\frac{\partial H_{B A}}{\partial \mathbf{k}} & \frac{\partial H_{B B}}{\partial \mathbf{k}}
\end{array}\right)
$$

Since $H_{A A}$ and $H_{B B}$ are independent of the wave vector $\mathbf{k}, \frac{\partial H_{A A}}{\partial \mathbf{k}}$ and $\frac{\partial H_{B B}}{\partial \mathbf{k}}$ are equal to zero. As a result, the velocity matrix is

$$
\mathbf{V}_{x}=\left(\begin{array}{cc}
0 & v_{f} \mathbb{1} \\
v_{f} \mathbb{1} & 0
\end{array}\right),
$$

here $\mathbb{1}$ is an $N \times N$ identical matrix and $v_{f}=\frac{3 b \alpha_{0}}{2 \hbar}$ is the Fermi velocity. After the action of the transformation matrix, $\mathscr{V}=U^{\dagger} V_{x} U$, the transferred velocity matrix is a diagonal block matrix, which is in the form of $\mathscr{V}=\mathscr{V}_{1} \oplus \mathscr{V}_{2} \oplus \cdots \oplus \mathscr{V}_{N}$. Each $\mathscr{V}_{j}$, a $2 \times 2$ matrix, is

$$
\mathscr{V}_{j}=\left(\begin{array}{cc}
0 & v_{f} \\
v_{f} & 0
\end{array}\right) .
$$

The unitary transformation matrix $\mathbf{U}$, diagonal block Green's function representation $\mathscr{G}$, and velocity operator $\mathscr{V}$ are now utilized to derive the analytical form of the dynamical conductivity of AANLG. 


\section{Dynamical Conductivity of AANLG}

The finite frequency conductivity is studied by using the Kubo formula. The conductivity is written in terms of the imaginary part of retarded current-current correlation function divided by frequency $\Omega$ as $\sigma_{\alpha \beta}=\frac{\operatorname{Im} \Pi_{\alpha \beta}\left(\Omega+\mathrm{i} 0^{\dagger}\right)}{\Omega}$, where $\Pi_{\alpha \beta}(\Omega)$ is also referred to as the polarization function. Furthermore, the polarization function can then be written in the bubble approximation as

$$
\Pi_{\alpha \beta}\left(i v_{m}\right)=e^{2} T \sum_{i \omega_{n}} \int \frac{d^{2} k}{(2 \pi)^{2}} \operatorname{Tr}\left[V_{\alpha} G\left(i \omega_{n}+i \mu_{m}, \mathbf{k}\right) V_{\beta} G\left(i \omega_{n}, \mathbf{k}\right)\right]
$$

where $V_{\alpha}$ is the velocity operator in the direction $\alpha=x$ or $y$ and $G\left(i \omega_{n}, \mathbf{k}\right)$ is the Green's function. With the spectral function representation

$$
G_{n m}(z)=\int_{-\infty}^{\infty} \frac{d \omega}{2 \pi} \frac{A_{n m}\left(\omega^{\prime}\right)}{z-\omega^{\prime}}
$$

the real part of the conductivity, at the zero temperature $T=0$, is expressed as

$$
\begin{aligned}
\sigma_{\alpha \beta}(\Omega) & =\frac{e^{2}}{2 \Omega} \int_{-\infty}^{\infty} \frac{d \omega}{2 \pi}[f(\omega-\mu)-f(\omega+\Omega-\mu)] \\
& \times \int \frac{d^{2} k}{(2 \pi)^{2}} \operatorname{Tr}\left[V_{\alpha} A(\omega+\Omega, \mathbf{k}) V_{\beta} A(\omega, \mathbf{k})\right],
\end{aligned}
$$

where $f(x)=1 /[\exp (x / T)+1]$ is the Fermi function and $\mu$ is the chemical potential. Following the aforementioned method, the AC conductivity for AANLG can be directly calculated by putting the $2 N \times 2 N$ Green's function representation (or spectral function representation) and $2 N \times 2 N$ velocity operator in Eq. $(23)$. The larger the Green's function (or spectral function representation) is, the more calculation tasks are.

To make less complex, we first utilize the relation, $\operatorname{Tr} M=\operatorname{Tr}\left[U^{\dagger} M U\right]$, invariant of the trace of a matrix (or operator) under a unitary transformation. Then, with a proper unitary transformation matrix, both the Green's function (or spectral function representation) and velocity operator $V_{\alpha}$ are reduced to the diagonal block matrices. As a result, the analytical form of the real part of the conductivity of AANLG can be easily accessible. The details are as below. First, by setting $M=V_{\alpha} G(\omega+\Omega, \mathbf{k}) V_{\beta} G(\omega, \mathbf{k})$, the trace of $M$ is $\operatorname{Tr} M=\operatorname{Tr}\left[U^{\dagger} V_{\alpha} G V_{\beta} G U\right]$. Then, insert the 
identical matrix $U U^{\dagger}=I$ between the velocity operator $V_{\beta}$ and Green's function $G$, and the result

$$
\operatorname{Tr} M=\operatorname{Tr}\left[\mathscr{V}_{\alpha} \mathscr{G} \mathscr{V}_{\beta} \mathscr{G}\right]=\operatorname{Tr} \mathscr{M}
$$

is acquired. $\mathscr{G}=U^{\dagger} G U$ is the unitary transformation of $G$ and it is related to the spectral function representation $\mathscr{A}$ in such a manner:

$$
\mathscr{G}_{m n}(z)=\int_{-\infty}^{\infty} \frac{d \omega}{2 \pi} \frac{\mathscr{A}_{m n}\left(\omega^{\prime}\right)}{z-\omega^{\prime}}
$$

$\mathscr{V}_{\alpha}=U^{\dagger} V_{\alpha} U$ is the unitary transformation of $V_{\alpha}$. Thirdly, after the unitary transformation, both the $\mathscr{V}$ and $\mathscr{A}$ are the diagonal block matrices. That is to say, the operator $\mathscr{M}=\mathscr{V}_{\alpha} \mathscr{A} \mathscr{V}_{\beta} \mathscr{A}$ is also a block diagonal matrix, such as, $\mathscr{M}=\mathscr{M}_{1} \oplus \mathscr{M}_{2} \oplus \mathscr{M}_{3} \oplus \cdots$. Each $\mathscr{M}_{j}=\mathscr{V}_{j, \alpha} \mathscr{G}_{j} \mathscr{V}_{j, \beta} \mathscr{G}_{j}$ is a two by two matrix. Finally, the relation $\operatorname{Tr}(\mathscr{M})=\operatorname{Tr}\left(\mathscr{M}_{1}\right)+\operatorname{Tr}\left(\mathscr{M}_{2}\right)+\operatorname{Tr}\left(\mathscr{M}_{3}\right)+\cdots$ is used to obtain the AC conductivity for AANLG

$$
\begin{aligned}
\sigma_{\alpha \beta}(\Omega) & =\frac{e^{2}}{2 \Omega} \int_{-\infty}^{\infty} \frac{d \omega}{2 \pi}[f(\omega-\mu)-f(\omega+\Omega-\mu)] \times \int \frac{d^{2} k}{(2 \pi)^{2}}\left[\operatorname{Tr} \mathscr{M}_{1}+\operatorname{Tr} \mathscr{M}_{2}+\operatorname{Tr} \mathscr{M}_{3}+\cdots\right] \\
& =\sum_{j=1}^{N} \frac{e^{2}}{2 \Omega} \int_{-\infty}^{\infty} \frac{d \omega}{2 \pi}[f(\omega-\mu)-f(\omega+\Omega-\mu)] \times \int \frac{d^{2} k}{(2 \pi)^{2}} \operatorname{Tr} \mathscr{M}_{j} \\
& =\sum_{j=1}^{N} \sigma_{j, \alpha \beta}(\Omega)
\end{aligned}
$$

It is shown that the AC conductivity of an AANLG is equal to the summation of the AC conductivity of each subsystem and $\sigma_{j, \alpha \beta}(\Omega)$ of each graphene-like layer is

$$
\begin{array}{r}
\sigma_{j, \alpha \beta}(\Omega)=\frac{e^{2}}{2 \Omega} \int_{-\infty}^{\infty} \frac{d \omega}{2 \pi}[f(\omega-\mu)-f(\omega+\Omega-\mu)] \\
\times \int \frac{d^{2} k}{(2 \pi)^{2}} \operatorname{Tr}\left[\mathscr{V}_{j, \alpha} \mathscr{A}_{j}(\omega+\Omega, \mathbf{k}) \mathscr{V}_{j, \beta} \mathscr{A}_{j}(\omega, \mathbf{k})\right]
\end{array}
$$

The AC conductivity of the AANLG can be analytically specified. As the $2 N \times 2 N$ Hamiltonian is decomposed into $N 2 \times 2$ reduced Hamiltonian matrices, the effective Hamiltonian of each subsystem is described as Eq. (13). Furthermore, the Green's function, spectral function representation and velocity operator associated with each subsystem are $2 \times 2$ matrices. Thus, 
the analytical form of AC conductivity of each graphene-like layer without ISOC is [52]

$$
\begin{array}{rr}
\sigma_{j, x x}(\Omega)= & \sigma_{\text {intra }}+\sigma_{\text {inter }}, \quad \text { without ISOC, } \\
\sigma_{\text {intra }}= & 4 \sigma_{0} \delta(\Omega)\left|\mu-\varepsilon_{j}\right| \Theta\left(\left|\mu-\varepsilon_{j}\right|\right), \\
\sigma_{\text {inter }}= & \sigma_{0} \Theta\left(\Omega-2\left|\varepsilon_{j}-\mu\right|\right),
\end{array}
$$

where intra and inter represent the contributions resulting from the intraband and interband transitions, respectively. With the ISOC taken into consideration, the analytical form of AC conductivity of each subsystem reads

$$
\sigma_{j, x x}(\Omega)=\sigma\left(\Omega,\left|\varepsilon_{j}-\mu\right|\right), \quad \text { with } \quad \text { ISOC }
$$

where $\sigma\left(\Omega,\left|\varepsilon_{j}-\mu\right|\right)$ is the conductivity for massive Dirac particles [62, 63], and it is expressed as

$$
\frac{\sigma(\Omega, \Upsilon)}{\sigma_{0}}=4 \frac{\Upsilon^{2}-\Delta^{2}}{\Upsilon} \delta(\Omega) \Theta(\Upsilon-\Delta)+\left[1+\left(\frac{2 \Delta}{\Omega}\right)^{2}\right] \Theta[(\Omega-2 \max (\Upsilon, \Delta))]
$$

The dependence of $\sigma_{j, x x}(\Omega)$ on the chemical potential $\mu, \varepsilon_{j}$ (or $\varepsilon_{\perp}$ ), and strength of ISOC is clearly revealed through the afore-presented formula.

The numerically calculated conductivity $\sigma_{x x}(\Omega)$ and the associated conductivity of each subsystem $\sigma_{j, x x}(\Omega)$ (denoted as sub-conductivity) of the AA-stacking trilayer graphene (TLG) are presented in Fig. 4. Both the intraband and interband transitions contribute to AC conductivity. A delta peak at frequency $\Omega=0$, caused by the intraband transition, is not shown here; that is, only the conductivity resulting from the interband transitions is shown. The conductivity $\sigma_{1, x x}(\Omega)$, $\sigma_{2, x x}(\Omega)$ and $\sigma_{3, x x}(\Omega)$ of the subsystems are illustrated in the dashed curves. AC conductivity of TLG are presented in the solid curve, which is $\sigma_{x x}(\Omega)=\sum_{J=1}^{N=3} \sigma_{1, j}(\Omega)$, the superposition of the AC conductivity of subsystems. According to Eqs. (26b) and 27b), the profile of each subconductivity $\sigma_{j, x x}(\Omega)=\sigma_{0} \Theta\left(\Omega-2\left|\varepsilon_{j}-\mu\right|\right)$ is governed by the step function $\Theta\left(\left|\varepsilon_{j}-\mu\right|\right)$. $\varepsilon_{j}$ related to TLG are $\varepsilon_{1}=-\sqrt{2 \alpha_{1}^{2}+V^{2}}, \varepsilon_{2}=0$, or $\varepsilon_{3}=\sqrt{2 \alpha_{1}^{2}+V^{2}}$. In the absence of the gated potential $(V=0)$ and at $\mu=0$, both $\sigma_{1, x x}$ and $\sigma_{3, x x}$ show the absorption edge at $\Omega / \alpha_{1}=\sqrt{2}$. $\sigma_{2, x x}(\Omega)$ contributes a constant background conductivity, which is equal to $\sigma_{0}$ (dashed curves 
in Fig. 4(a)). AC conductivity of TLG (solid curve Fig. 4(a)) at high frequency is equal to a constant value, three times of $\sigma_{0}$. At $\mu=0.1 \alpha_{1}, \sigma_{1, x x}, \sigma_{2, x x}$ and $\sigma_{3, x x}$ in Fig. 3(b)) show step edges at frequencies $\Omega / \alpha_{1}=\sqrt{2}+0.1,0.2$ and $\sqrt{2}+0.1$. As a result, there are three steps in the AC conductivity (solid curve in Fig. 4(b)). In the application of the gated potential $V=0.4 \alpha_{1}$, the absorption edges of $\sigma_{1, x x}$ and $\sigma_{3, x x}$ occur at $\Omega=(\sqrt{2.16}+0.1) \alpha_{1}$ and $\Omega=(\sqrt{2.16}-0.1) \alpha_{1}$ (Fig. 4 (c)). The intrinsic SOC $\left(\Delta=0.1 \alpha_{1}\right)$ enhances the strength of sub-conductivity $\sigma_{2, x x}$ in the region $0.2<\Omega / \alpha_{1}<0.6$. For comparison, the solid curves in Figs. 4(a)-4(d) are plotted in Fig. 4(e). The characteristics of $\sigma_{x x}(\Omega)$ of TLG are dependent on $\mu, V$, and strength of ISOC.

The alternation of layer number $N$ has a great influence on AC conductivity of an AANLG. Figure 5 displays $\sigma_{x x}(\Omega)$ and the associated $\sigma_{j, x x}(\Omega)$ of the AA-stacking quad -layer graphene (QLG) . At high frequency, AC conductivity of QLG illustrates a constant value, which is equal to four times of $\sigma_{0}$. There are two steps in the AC conductivity of QLG at $V=0$ (the solid blue curve in Fig. 5(a)). The location of the absorption edge of each sub-conductivity $\sigma_{j, x x}(\Omega)$ is controlled by the step function $\Theta\left(\left|\varepsilon_{j}-\mu\right|\right)$. The gated-potential-dependent energy dispersions $\varepsilon_{j}$ related to QLG are $\varepsilon_{ \pm \pm}= \pm \sqrt{\mathrm{B} \pm \sqrt{\mathrm{B}^{2}-\mathrm{C}}}$, where $\mathrm{B}=\frac{3 \alpha_{1}^{2}}{2}+\frac{5 V^{2}}{4}$ and $\mathrm{C}=\alpha_{1}^{4}+\frac{3}{4} \alpha_{1}^{2} V^{2}+\frac{9}{16} V^{4}$. In the absence of the gated potential $(V=0), \varepsilon_{++}=-\varepsilon_{-+}=2.618 \alpha_{1}$ and $\varepsilon_{+-}=-\varepsilon_{--}=$ $0.382 \alpha_{1}$. The first and second absorption edges appear at $\Omega / \alpha_{1}=0.76$ and $\Omega / \alpha_{1}=5.2$ (Fig. 5(a)). $\sigma_{1, x x}(\Omega)\left(\sigma_{3, x x}(\Omega)\right)$ is identical to $\sigma_{2, x x}(\Omega)\left(\sigma_{4, x x}(\Omega)\right)$. At $\mu=0.3 \alpha_{1}$, absorption edges occur at $\Omega / \alpha_{1} \approx 0.2,1.4,4.6$, and 5.8. AC conductivity features four steps (the solid cyan curve in Fig. 5(b)). The gated potential $V=0.3 \alpha_{1}$ modifies $\varepsilon_{++}=2.881 \alpha_{1}$ and $\varepsilon_{+-}=0.344 \alpha_{1}$ and changes the locations of step edges (Fig. 5(c)). The intrinsic SOC $\Delta=0.1 \alpha_{1}$ enhances the weight of AC conductivity around $\Omega / \alpha_{1} \approx 0.2$. The solid curves in Figs. 5(a)-5(d) are plotted in Fig. 5(e) to illustrate that the effects caused by the alteration of $N, \mu, V$, and strength of ISOC on $\sigma_{x x}(\Omega)$ of an AANLG are easily and clearly revealed through the analytical formula.

\section{Conclusions}

In this work, we propose an analytical model to derive the exact energy spectrum and dynamical conductivity in an AANLG in the presence of a bias voltage and spin-orbital coupling at the same footing. First, a proper transformation matrix is built and used to transform the $2 N \times 2 N$ 
tight-binding Hamiltonian matrix of an AANLG into $N 2 \times 2$ diagonal block matrices. Then, an AANLG is reduced to $N$ graphene-like layers. Thus, the exact energy spectrum of a graphenelike layer is $E=\varepsilon_{\perp} \pm \varepsilon_{\|} \cdot \varepsilon_{\perp}$, the effective on-site energy of the graphene-like layer, is controlled by the interlayer interaction, gated potential, and layer number. $\varepsilon_{\|}$is the energy spectrum of a monolayer graphene with SOC. Furthermore, we analytically study the dynamical conductivity of an AANLG, which is shown to be the sum of the dynamical conductivity of $N$ graphene-like layers with/without SOC. The dependence of the dynamical conductivity of each graphene-like layer on the chemical potential, $\varepsilon_{\perp}$, and the strength of SOC is clearly demonstrated. Above all, our model can efficiently and exactly acquire the energy spectrum and dynamical conductivity in a gated AANLG with SOC.

\section{Acknowledgements}

The author gratefully acknowledges the support of the Taiwan National Science Council under the Contract Nos. NSC 102-2112-M-165-001-MY3.

\section{References}

[1] K. S. Novoselov, A. K. Geim, S. V. Morozov, D. Jiang, Y. Zhang, S. V. Dubonos, I. V. Grigorieva and A. A. Firsov, Science, 2004, 306, 666-669.

[2] C. Berger, Z. Song, T. Li, X. Li, A. Y. Ogbazghi, R. Feng R, Z. Dai, A. N. Marchenkov, E. H. Conrad, P. N. First and Walt A. de Heer, J. Phys. Chem. B, 2004, 108, 19912-19916.

[3] M. Katsnelson, K. Novoselov and A. Geim, Nat. Phys., 2006, 2, 620-625.

[4] C. Beenakker, Colloquium: Andreev reflection and Klein tunneling in graphene, Rev. of Mod. Phys., 2008, 80, 1337.

[5] Z. Jiang, E. A. Henriksen, C. Tung, Y.-J. Wang, M. E. Schwartz, M. Y. Han, P. Kim and H. L. Stormer, Phys. Rev. Lett., 2007, 98, 197403.

[6] E. Hwang and S. D. Sarma, Phys. Rev. B, 2007, 75, 205418. 
[7] K.I. Bolotin, K.J. Sikes, Z. Jiang, M. Klima, G. Fudenberg, J. Hone, P. Kim and H. L. Stormer, Solid State Commun., 2008, 146, 351-5.

[8] X. Du, I. Skachko, A. Barker and E. Y. Andrei, Nat. Nanotech. 2008, 3, 491-495.

[9] R. R. Nair, P. Blake, A. N. Grigorenko, K. S. Novoselov, T. J. Booth, T. Stauber, N. M. R. Peres and A. K. Geim, Science ,2008, 320, 1308-1308.

[10] C. Gomez-Navarro, R. T. Weitz, A. M. Bittner, M. Scolari, A. Mews, M. Burghard and K. Kern, Nano. Lett. 2007, 7, 3499-3503.

[11] M. Katsnelson, Eur. Phys. J. B, 2006, 51, 157-160.

[12] K. S. Kim, Y. Zhao, H. Jang, S. Y. Lee, J. M. Kim, K. S. Kim, J. H. Ahn, P. Kim, J. Y. Choi and B. H. Hong, Nature, 2009, 457, 706-710.

[13] L. Malard, M. Pimenta, G. Dresselhaus and M. Dresselhaus, Phys. Rep., 2009, 473, 51-87.

[14] T. Stauber, N. Peres and A. Geim, Phys. Rev. B, 2008, 78, 085432.

[15] K. I. Bolotin, F. Ghahari, M. D. Shulman, H. L. Stormer and P. Kim, Nature, 2009, 462, 196-199.

[16] A. A. Balandin, S. Ghosh, W. Bao, I. Calizo, D. Teweldebrhan, F. Miao and C. N. Lau, Nano. Lett. 2008, 8, 902-907.

[17] S. D. Sarma, S. Adam, E. Hwang and E. Rossi, Rev. Mod. Phys., 2011, 83, 407.

[18] X. Wang, L. Zhi and K. Mullen, Nano. Lett., 2008, 8, 323-327.

[19] F. Wang, Y. Zhang, C. Tian, C. Girit, A. Zettl, M. Crommie and Y. R. Shen, Science, 2008, 320, 206-209 .

[20] P. Avouris, Nano letters, 2010, 10, 4285-4294.

[21] F. Bonaccorso, Z. Sun, T. Hasan and A. Ferrari, Nature Photonics, 2010, 4, 611-622.

[22] C. Dean, A. Young, I. Meric, C. Lee, L. Wang, S. Sorgenfrei, K. Watanabe, T. Taniguchi, P. Kim and K. Shepard, Nat. nanotech, 2010, 5, 722-726. 
[23] F. Schwierz, Nat. Nanotech., 2010, 5,487-496.

[24] Q. He, S. Wu, Z. Yin and H. Zhang, Chem. Sci., 2012, 3, 1764-1772.

[25] P. Avouris and C. Dimitrakopoulos, Mater. Today, 2012, 15 (3), 86-97.

[26] S. Latil and L. Henrard, Phys. Rev. Lett., 2006, 97, 036803.

[27] D. Graf, F. Molitor, K. Ensslin, C. Stampfer, A. Jungen, C. Hierold and L. Wirtz, Nano. lett., 2007, 7, 238-242.

[28] J. Hass, W. De Heer and E. Conrad, J. Phys.: Condens. Matt., 2008, 20, 323202.

[29] J. Nilsson, A. C. Neto, F. Guinea and N. Peres, Phys . Rev. B, 2008, 78 ,045405.

[30] H. Min and A. MacDonald, Phys. Rev. B, 2008, 77, 155416.

[31] C. H. Lui, Z. Li, Z. Chen, P. V. Klimov, L. E. Brus and T. F. Heinz, Nano. lett., 2010, 11, 164-169.

[32] T. Ohta, A. Bostwick, T. Seyller, K. Horn and E. Rotenberg, Science, 2006, 313, 951-954.

[33] E. McCann and V. I. Fal'ko. Phys. Rev. Lett. 2006. 96, 086805.

[34] A. Ferrari, J. Meyer, V. Scardaci, C. Casiraghi, M. Lazzeri, F. Mauri, S. Piscanec, D. Jiang, K. Novoselov and S. Roth, Phys. Rev. Lett., 2006, 97, 187401.

[35] E. V. Castro, K. Novoselov, S. Morozov, N. Peres, J. L. Dos Santos, J. Nilsson, F. Guinea, A. Geim and A. C. Neto, Phys. Rev. Lett., 2007, 99, 216802.

[36] D. Abergel and V.I. Fal'ko, Phys. Rev. B, 2007, 75, 155430.

[37] J. B. Oostinga, H. B. Heersche, X. Liu, A. F. Morpurgo and L. M. Vandersypen, Nature materials, 2007, 7, 151-157.

[38] L. Zhang, Z. Li, D. N. Basov, M. Fogler, Z. Hao and M. C. Martin, Phys. Rev. B, 2008, 78, 235408.

[39] Y. Zhang, T. Tang, C. Girit, Z. Hao, M. C. Martin, A. Zettl, M. F. Crommie, Y. R. Shen and F. Wang, Nature, 2009, 459, 820-823. 
[40] C. P. Chang, RSC Adv., 2014, 4, 32117-32126.

[41] M. Craciun, S. Russo, M. Yamamoto, J. B. Oostinga, A. Morpurgo and S. Tarucha, Nat. nanotech., 2009, 4, 383-388.

[42] M. Koshino and E. McCann, Phys. Rev. B, 2009, 80, 165409.

[43] F. Zhang, B. Sahu, H. Min and A. H. MacDonald, Phys. Rev. B, 2010, 82, 035409.

[44] S. Yuan, R. Roldan and M. I. Katsnelson, Phys. Rev. B, 2011, 84, 125455.

[45] J. K. Lee, S. C. Lee, J. P. Ahn, S. C. Kim, J. I. Wilson and P. John, J. Chem. Phys., 2008, 129,234709 .

[46] J. Soltys and J. Piechota. J. Appl. Phys., 2011, 109, 093523.

[47] Z. Liu, K. Suenaga, P. J. Harris and S. Iijima, Phys. Rev. Lett., 2009, 102, 015501.

[48] Y. H. Xu, X. W. Li and J. M. Dong, Nanotechnology, 2010, 21, 065711.

[49] Y. H. Ho, J. Y. Wu, R. B. Chen, Y. H. Chiu and M. F. Lin, Appl. Phys. Lett., 2010, 97, 101905.

[50] C. P. Chang, J. Appl. Phys., 2011, 110, 013725.

[51] M. F. Lin, Y. C, Chuang and J. Y. Wu, Phys. Rev. B, 2012, 86, 125434.

[52] C. J. Tabert and E. J. Nicol, Phys. Rev. B, 2012, 86, 075439.

[53] Y. Mohammadi, R. Moradian and F. S, Tabar, Solid State Commun. 2014, 193, 1-5 .

[54] C. P. Chang, J. Phys. Chem. C, 2012, 116, 22073-22077.

[55] C. P. Chang, Carbon, 2013, 61, 209-215.

[56] C. L. Kane and E. J. Mele, Phys. Rev. Lett., 2005, 95, 146802.

[57] P. Rakyta, A. Kormanyos, and J. Cserti, Phys. Rev. B, 2010, 82, 113405.

[58] J. C. Charlier, J. P. Michenaud and X. Gonze, Phys. Rev. B, 1992, 46, 4531-4539. 
[59] S. Konschuh, M. Gmitra, D. Kochan, and J. Fabian, Phys. Rev. B, 2012, 85, 115423.

[60] A. Kormanyos and G. Burkard, Phys. Rev. B , 2013, 87, 045419.

[61] R. van Gelderen, R. Olsen and C. Morais Smith, Phys. Rev. B, 2013, 88, 115414.

[62] V. P. Gusynin, S. G. Sharapov and J. P. Carbotte, Phys. Rev. Lett., 2006, 96, 256802.

[63] W. K. Tse and A. H. MacDonald, Phys. Rev. Lett., 2010, 105, 057401. 


\section{Figure Captions}

FIG. 1. The geometric structure of the AA-stacking multilayer graphene and the intralayer and interlayer interactions.

FIG. 2. Calculated energy dispersions around the Dirac point $\mathbf{K}$ of the AA-stacking bilayer graphene for different gateed potential $V$, intrinsic $\operatorname{SOC} \Delta$, and Rashba SOC $\lambda_{R}$. The dashed curves in the inset: $\left(V, \Delta, \lambda_{R}\right)=(0,0,0)$; red solid curves: $(0.4,0,0) \alpha_{1}$; blue solid curves: $(0.4,0.1,0) \alpha_{1}$ ; green solid curves: $(0.4,0.0,0.05) \alpha_{1}$.

FIG. 3. Calculated energy dispersions of the AA-stacking trilayer graphene for different $V, \Delta$, and $\lambda_{R}$. The dashed curves in the inset: $\left(V, \Delta, \lambda_{R}\right)=(0,0,0)$; red solid curves: $(0.4,0,0) \alpha_{1}$; blue solid curves: $(0.4,0.1,0) \alpha_{1}$; green solid curves: $(0.4,0.1,0.05) \alpha_{1}$.

FIG. 4. AC conductivity $\sigma_{x x}$ (solid curves) and sub-conductivity $\left(\sigma_{1}, \sigma_{2}, \sigma_{3}\right)$ (dashed curves) of the AA-stacking trilayer graphene for different $V, \Delta$, and $\mu$ are presented. (a) $(V, \Delta, \mu)=$ $(0,0,0)$. (b) $(V, \Delta, \mu)=(0,0,0.1) \alpha_{1}$. (c) $(V, \Delta, \mu)=(0.4,0,0.1) \alpha_{1}$. (d) $(V, \Delta, \mu)=(0.4,0.1,0.1) \alpha_{1}$. The solid curves in (a)-(d) are plotted in (e).

FIG. 5. Same plot as Fig.3 but for AA-stacking quad-layer graphene. (a) $(V, \Delta, \mu)=(0,0,0)$. (b) $(V, \Delta, \mu)=(0,0,0.3) \alpha_{1}$. (c) $(V, \Delta, \mu)=(0.3,0,0.3) \alpha_{1}$. (d) $(V, \Delta, \mu)=(0.3,0.1,0.3) \alpha_{1}$. The solid curves in (a)-(d) are displayed in (e). 


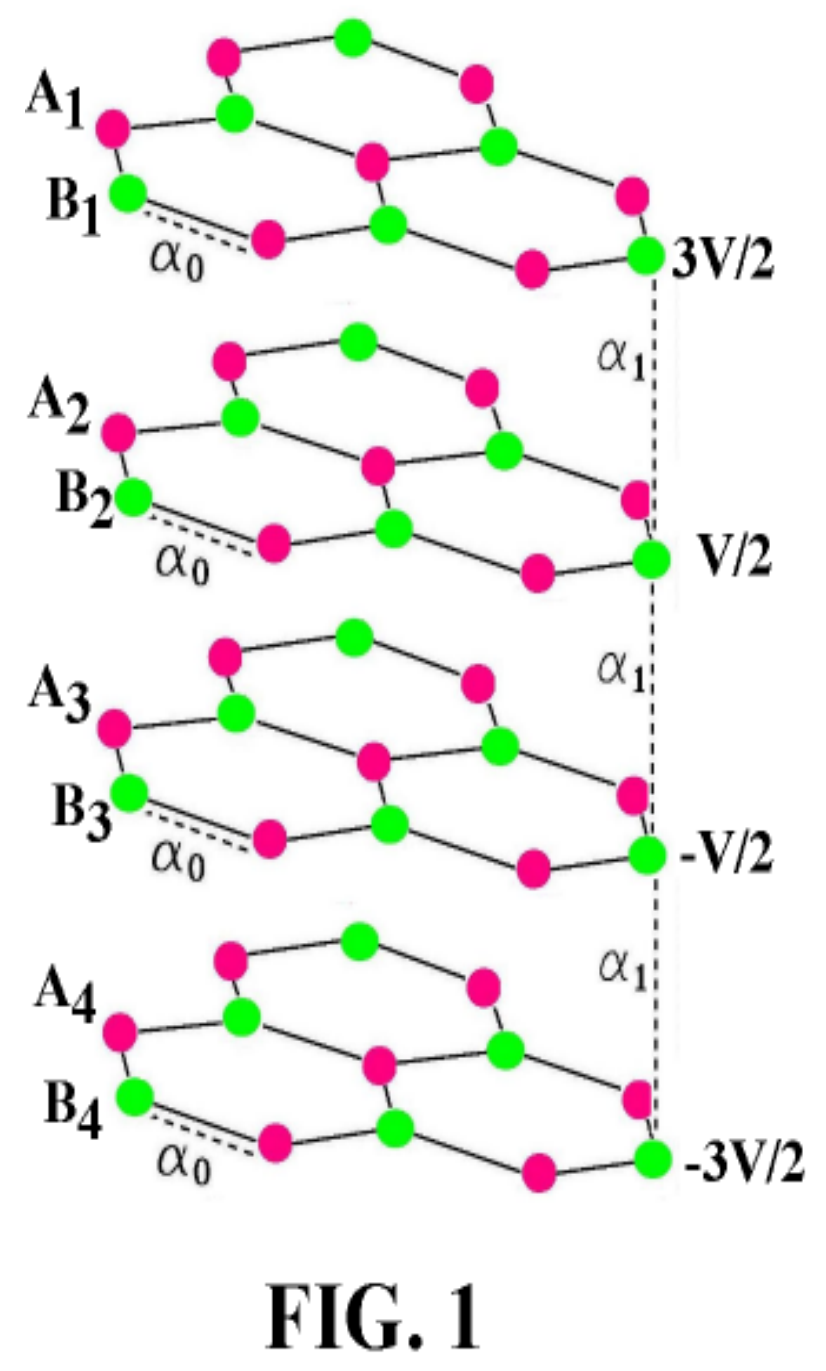




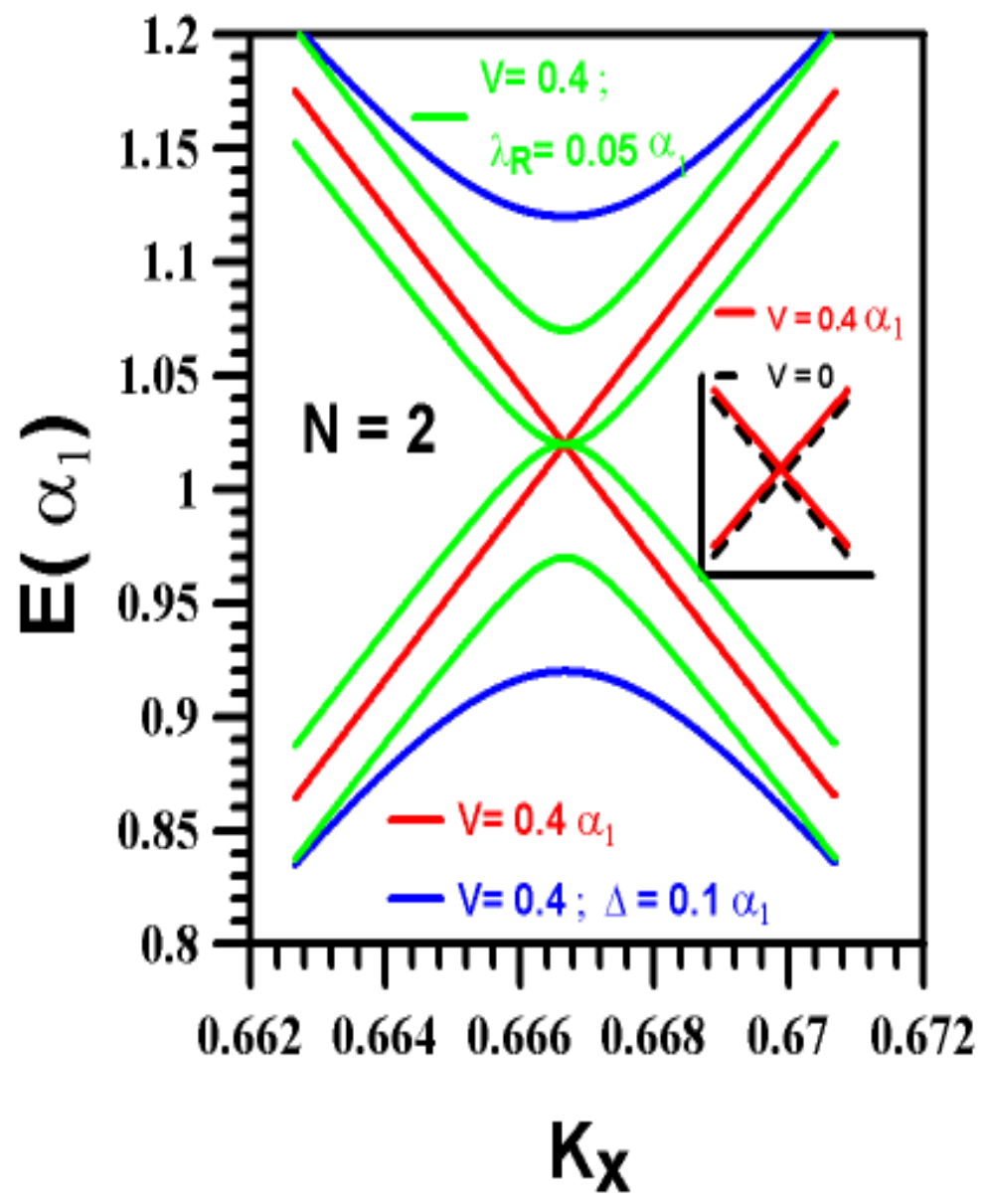

FIG. 2 


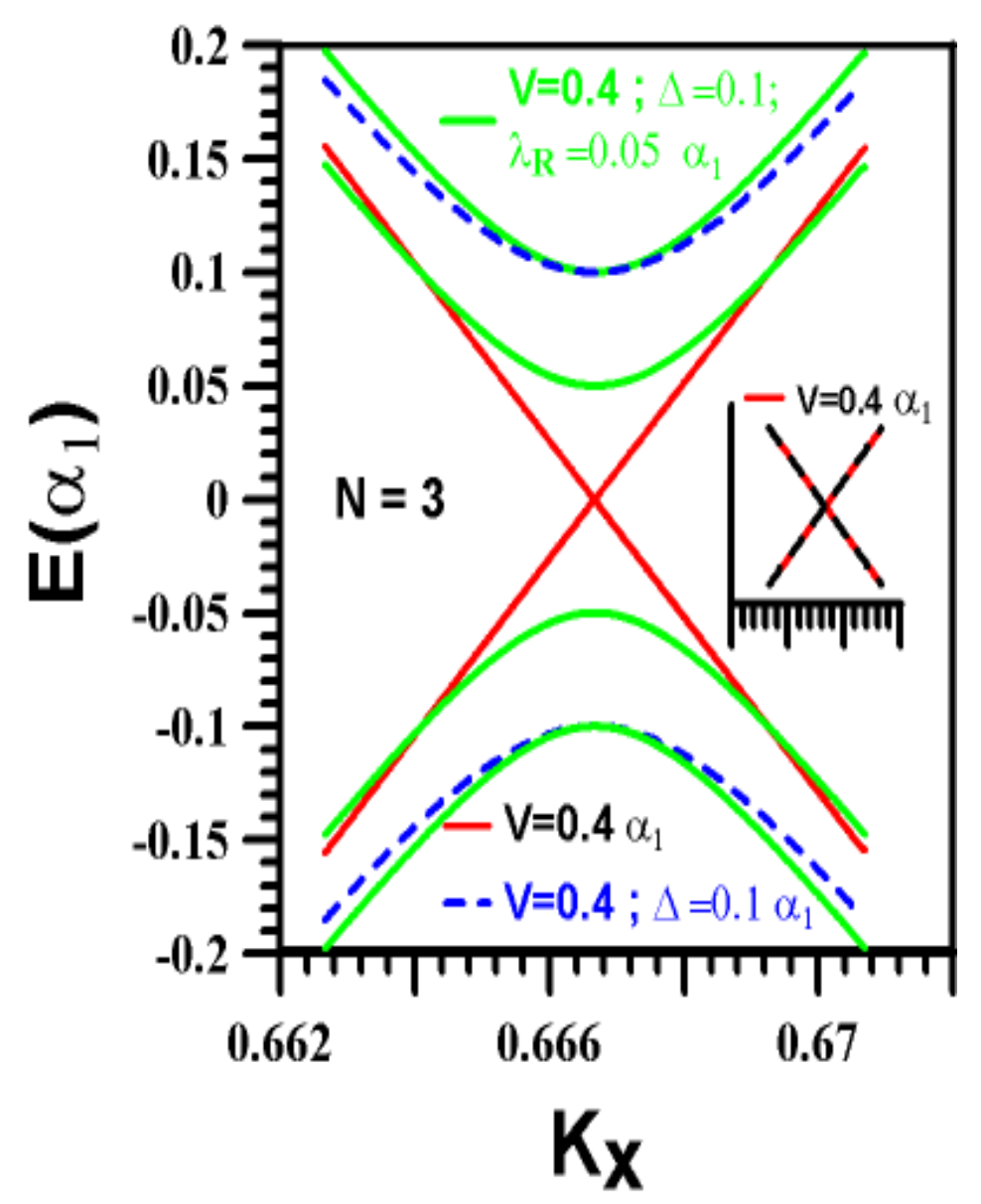

FIG. 3 


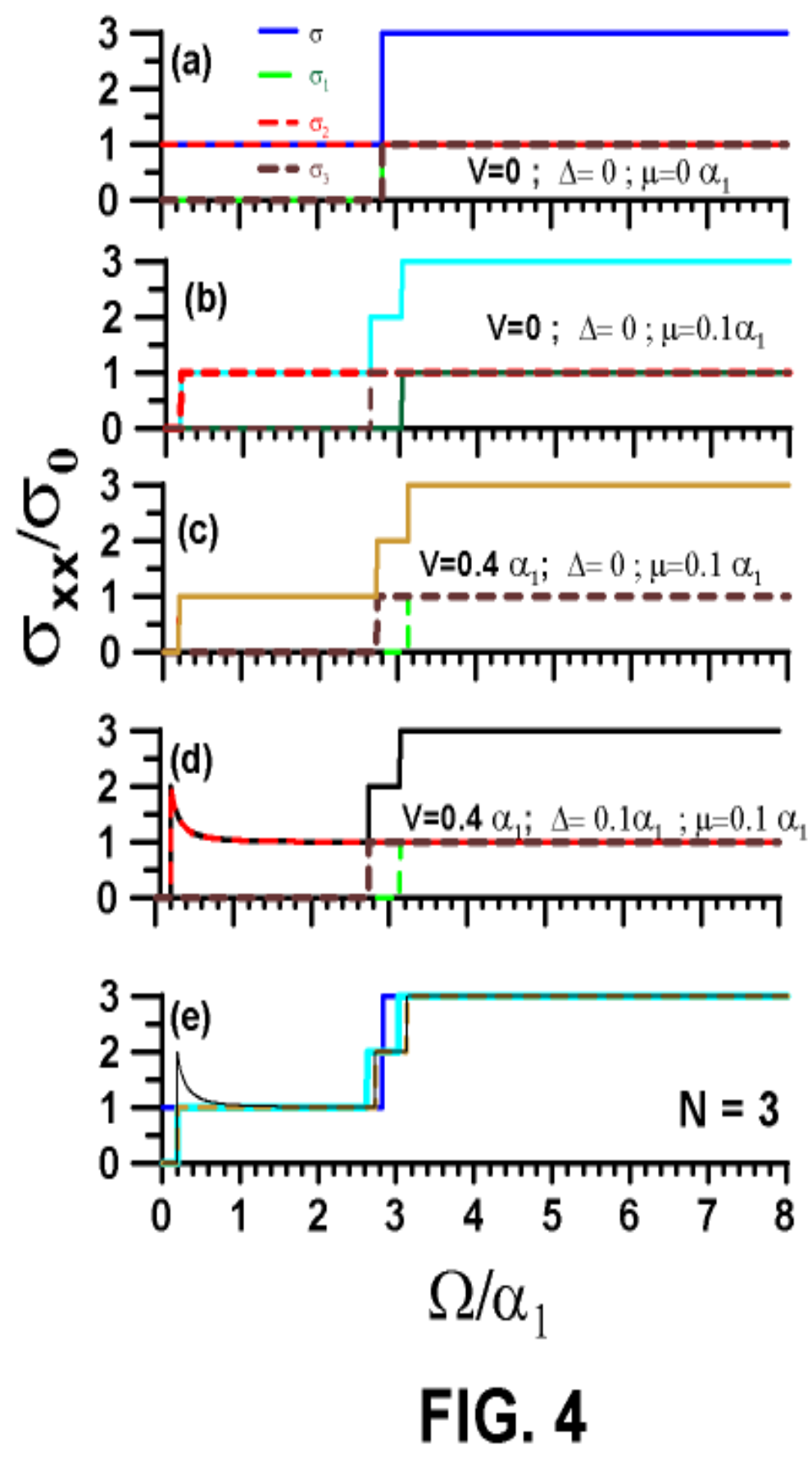




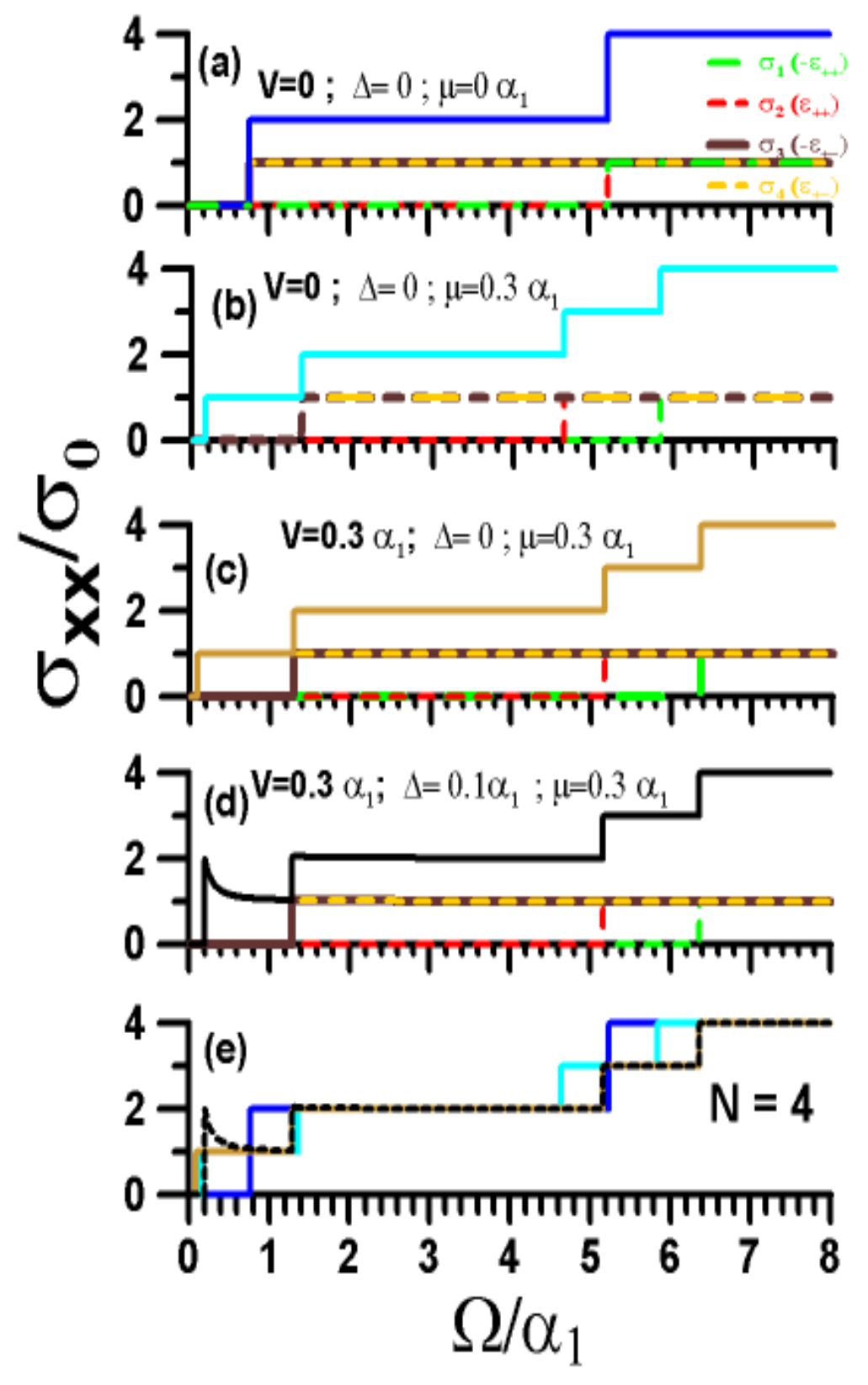

FIG. 5 